FRANK M. SCHUSTER

\title{
Die verborgene Stadt. Die Wiederentdeckung der polyethnischen Vergangenheit der Stadt Łódź
}

\begin{abstract}
Niniejszy artykuł podejmuje kwestię, w jaki sposób Łódź, niegdyś wielokulturowe miasto przemysłowe, jest $\mathrm{w}$ regionie $\mathrm{i}$ po za nim postrzegana, interdyscyplinarnie badana i opisywana. W szczególności tekst koncentruje się na roli, jaką łódzka germanistyka odegrała na przestrzeni ostatnich dwudziestu lat w procesie ponownego odkrywania wspólnej dla Niemców, Polaków i Żydów przeszłości.
\end{abstract}

Der Artikel geht der Frage nach, wie die ehemals multikulturelle Industriestadt Łódź in der Region (aber auch darüber hinaus) wahrgenommen und wie darüber (interdisziplinär) geforscht und geschrieben wird. Der Schwerpunkt liegt dabei vor allem auf der Rolle der Łódźer Germanistik bei der Wiederentdeckung der deutsch-polnisch-jüdischen Vergangenheit in den letzten zwanzig Jahren.

The article deals with the perception of the former multicultural industrial city of Łódź in the region itself (and beyond), how research on this topic looks like, and how one writes about it. The text focuses especially on the role of the German Studies in Łódź in process of rediscovering, in the last twenty years, the city's German-Polish-Jewish past.

Fast ist man versucht, Lodz ${ }^{1}$ für eine jener verborgenen Städte ITALO CALVINOs (2007) zu halten, deren Bild dieser in seinem Buch Die unsichtbaren Städte so meisterlich malt. Denn auch Lodz gehört zu jenen unsichtbaren, verborgenen Städten, deren Geschichte mehr in Erzählungen und Mythen als in

\footnotetext{
Den zeitgenössisch, wie gegenwärtig am Ort üblichen Gebrauch aufgreifend, verwende ich im Weiteren, weil der vorliegende Text auf Deutsch verfasst wurde, die deutsche Form des Stadtnamens in der dort selbst bis 1939 üblichen Form: Lodz analog z. B. zu Warschau. Nur in den Fällen in denen explizit die ethnisch weitgehend homogen polnische Stadt nach 1945 gemeint ist, gebrauche ich die polnische Form Łódź. Das hat ausschließlich praktische Gründe. Eine weitere Ausdifferenzierung hätte nämlich unter anderem zur Folge dass ich die jüdische Schreibweise ebenfalls berücksichtigen müsste. Diese lautete im Original לודזש, d.h. in der heutzutage wissenschaftlich üblichen - aus dem Englischen stammenden - Transkription Lodzh. Um keine zusätzliche Verwirrung zu stiften, habe ich mich daher weitgehend auf die Verwendung einer Schreibweise beschränkt. Aus denselben pragmatischen Gründen spreche ich auch vom Ghetto Lodz, obwohl ich genauso gut Getto Łódź sagen könnte, da es bisher in der internationalen Forschung terminologisch zu keiner Einigung gekommen ist. Streng genommen müsste es ohnehin Ghetto Litzmannstadt heißen.
} 
Frank M. Schuster

der Realität weiterlebt. 1793 noch abseits gelegenes verschlafenes Provinzstädtchen, in dem 190 Menschen lebten, sollte es zur am schnellsten wachsenden Stadt im Europa des 19. Jhd.s werden, mit einer halben Million Einwohnern kurz vor dem Ersten Weltkrieg. „,... Diesen] beispiellose[n] Aufstieg von einem unbedeutenden Nest zur bedeutendsten Industriemetropole Ostmitteleuropas“ (SCHLÖGEL 1996:127) verdankt Lodz ,[a]ls einzige Großstadt im europäischen Kulturbereich [...] generationenlang Angehörigen verschiedener Nationalitäten, unter denen Deutsche, Juden und Polen stets insgesamt dominierten, [wobei] die zahlenmäßige Proportion der drei Gruppen innerhalb der Gesamtbevölkerung sich aber ständig veränderte. ${ }^{\text {“2 }}$ Letztlich sollte aber auch die Entwicklung zu dem kongresspolnischen Textilindustriezentrum nicht dazu führen, dass Lodz und seine Bewohner außerhalb der Region die gebührende Beachtung erhielten. Lodz blieb unsichtbar. Wenn etwas unsichtbar, d. h. nicht zu sehen ist, so kann das mehrere Gründe haben: Entweder der Betrachter ist blind oder er sieht nichts, weil er nicht weiß, dass es etwas zu sehen gibt. Man nimmt nur das wahr, was man kennt. Unbekanntes übersieht man leicht, ebenso wie Verdecktes. Spätere Ereignisse, Erlebnisse und Erfahrungen können frühere überlagern. Glaubt man zudem bereits zu wissen, was einen erwartet, so ist man oft nicht in der Lage, wirklich das zu sehen, was man vor sich hat. Vorgefasste Bilder prägen die Wahrnehmung. Etwas, was man nicht oder nicht mehr wahrnimmt, etwas, was man aus dem Blick verliert, wird leicht vergessen. Aus den Augen, aus dem Sinn - heißt es nicht umsonst.

All dies trifft auf Lodz zu. Einer der Gründe dafür liegt darin, dass der kometenhafte Aufstieg der Stadt, der sich unter russischer Herrschaft vollzog, zu großen Teilen von deutschen und jüdischen Fabrikanten und Händlern initiiert und forciert, aber überwiegend von polnischen Arbeitern getragen wurde. Die polnische Gesellschaft dagegen war ländlich, von Bauern und Adel dominiert. Daher war eine Beschäftigung mit Handwerk und Handel in adligen Kreisen seit der Zeit der Adelrepublik verpönt. Sie war - so JÖRG GEBHARD (2006:9f.) -

[...] durch die kulturell normsetzende Adelselite traditionell negativ konnotiert, ein Verdikt, das über das Gedächtnis der Adelsrepublik als Nukleus polnischer Nationalidentität auch über die Zäsur der Teilungen transportiert und durch die

2 Siehe Jürgen Hensels Konferenzbericht zu der internationalen, interdisziplinären Konferenz „Gruppenbeziehungen in einer multiethnischen Stadtgesellschaft: Polen, Deutsche und Juden in Lodz im 19. und 20. Jahrhundert (bis 1939)“ vom 19.22. Oktober 1995 in Łódź, erschienen 1996 im 2. Bulletin des Deutschen Historischen Instituts Warschau, auch im Internet abrufbar unter http://www.dhi.waw.pl/ bulletin2/bd2_07.htm (1.9.2006). 
Erfahrung der Industrialisierung durch die polnische Öffentlichkeit in seiner Wirksamkeit eher gestärkt als geschwächt wurde. Das [...] negative Imago Lodzs gehört in diesen Problemzusammenhang. Gleichzeitig wurden auch die Akteure der Industrialisierung selbst, die Unternehmer, von Literatur und Publizistik gegen das Idealbild des Szlachtizen gespiegelt. Dadurch entstand ein ebenfalls düsteres Zerrbild, welches von der sozialistischen Kapitalismuskritik wieder aufgegriffen wurde und bis in die jüngste Vergangenheit überdauert hat.

„Lodz erscheint von Warschau aus fast wie ein Vorort, keine Stadt von eigenem Gewicht“, meinte KARL SCHLÖGEL (1996:127) bereits 1996 in einem seiner Reisefeuilletons. Große nationale Traditionen, an die eine polnische Memoria hätte anknüpfen können, fanden sich gerade hier nicht, wie ANDREAS R. HOFMANN (2002:238) ebenfalls feststellt: „Denn für die polnische nationalkulturelle Öffentlichkeit war die Stadt schlicht historisch bedeutungslos, weil sie niemals Schauplatz eines jener für die nationale Identität damals zentralen politischen und kulturellen ,Ereignisse " gewesen war." Lodz ist von Anfang an für die Mehrheit der Polen die ungeliebte, die ignorierte Stadt, denn sie war, wie GEBHARD (2006:6) zu Recht hervorhebt, eine Ausnahme:

Eine Industriestadt im besten und im schlechtesten Sinne des Wortes, in der anfangs vor allem Deutsche und polnische Juden ein Zentrum der europäischen Tuchfabrikation aus dem Boden stampften und eine städtische Konstellation kreierten, die es in einer solchen Form weder zuvor noch danach gegeben hat. Eine Stadt, die wegen ihrer Einzigartigkeit mit einem zweifelhaften Image zu kämpfen hat - bis heute. Allein der bekannte Lodz-Roman Das gelobte Land (Ziemia obiecana) des polnischen Schriftstellers Reymont (1899) und dessen Verfilmung durch den Oskarpreisträger Andrzej Wajda (1974) ist eine nicht zu überschätzende, sich landesweit auswirkende PR-Katastrophe für die Stadt.

Der Roman wurde - so auch HoFMANN (2002a:240) - ,[e]in stilbildender Topos“, denn er ,war nicht die erste, jedoch die bekannteste und nachhaltig wirkungsvollste literarische Behandlung des Themas Lodz. Bis heute hat sich das Buch seinen Ruf als Lodz-Roman schlechthin bewahrt.“ Mehr noch: Der Roman war und ist - zumindest was die polnische Sicht auf die Stadt angeht wahrnehmungsdominierend, wie ein Blick auf die nach dem Buch WŁADYSŁAW STANISŁAW REYMONTs (1899) entstandenen Reportagen zeigt (vgl. z.B. BARTKIEWICZ 1911; STOLARZEWCZ 1935; FREJDLICH 1973; WILMAŃSKI 1993; GÓRECKI 1998). Das heutige Bild von Lodz ist somit weniger durch historisches Wissen als vielmehr von Mythen geprägt, wie der Lodzer Journalist und Schriftsteller JACEK INDELAK (1997:2716, 2721) konstatierte:

Lodz ist geradezu überwuchert mit Mythen. Dem Mythos vom ,Gelobten Land“ für wenige und der schlechten Stadt für viele, den Wladyslaw Reymont in seinem gleichnamigen Roman 1899 beschwört, folgt der Gründermythos, der den rasan- 
Frank M. Schuster

\begin{abstract}
ten Aufbau von kapitalistischen Unternehmen, von Aktiengesellschaften, von Börsenspekulationen, hohen Gewinnen und schnellem Reichtum verklärt. Die Volksrepublik verbreitete wirkungsvoll den Mythos vom ,roten Lodz' als Wiege des einheimischen Kommunismus und interpretierte dabei die Geschichte ganz in ihrem Sinne. Und schließlich ein relativ junger Mythos. Lodz als Stadt der vier Kulturen, Religionen, Völker: Polen, Deutsche, Juden, Russen. Doch eine Vielvölkerstadt wie Lodz war in Polen bis 1939 normal. [...] Das Lodz von damals gibt es nicht mehr. Die Nazis haben es liquidiert. Für immer. Der Kommunismus legte einen Mantel des Schweigens über die Welt von einst.
\end{abstract}

Erst in den letzten Jahren beginnt in der Stadt selbst langsam eine Auseinandersetzung mit der Vergangenheit, denn „Lodz versucht heute, sein Gedächtnis wiederzufinden, sein kollektives Gedächtnis. Dabei bedient die Stadt sich der Mythologie“" (INDELAK 1997:2721, s. auch STROBEL 1993). Eine mythisierende, lokale Sicht auf die Stadt und ihre Geschichte auf der einen sowie eine immer noch überwiegend negative polnische Wahrnehmung von Lodz, zumindest außerhalb der Stadt selbst, auf der anderen Seite prägen die Wahrnehmung - sofern die Stadt überhaupt wahrgenommen wird. Doch die Dichotomie zwischen verklärender lokaler Selbstwahrnehmung und negativer Fremdwahrnehmung auf polnischer Seite ist nur ein Teil des Stadtbildes. Schließlich existierte oder existiert noch die Wahrnehmung der anderen ehemals in Lodz lebenden Ethnien, allen voran der Juden und Deutschen.

Jüdischerseits ist die Stadtgeschichte weitgehend durch die Geschichte jenes Ghettos, das die Deutschen 1940 im Norden der Stadt errichteten, und die Shoah überlagert, zumindest was das Interesse vor allem der polnisch-, deutsch- oder englischsprachigen Öffentlichkeit angeht (vgl. z.B. ALBERTI 2006; Löw 2006). Die Stadt vor der Vernichtung findet sich allerdings in literarischen Werken vor allem aus den 20er und 30er Jahren (RABON 1928, 1934; I. J. SINGER 1936) sowie zum Teil auch in den Erinnerungen, Aufzeichnungen und Romanen jener wieder, die die Katastrophe überlebten (vgl. z.B. TRUNK 1944-1953; SHPIGL 1966; RUBINSTEIN 1973; BLOCH 1981; MostowiCZ 1988; AKSZTAJN 1996).

In Deutschland ist die Erinnerung an das deutsche Lodz vor 1939 ins Liedgut abgesunken, wie Ethnologen und Ethnographen es nennen, denn in Deutschland assoziiert die breite Öffentlichkeit mit dem Stadtnamen bestenfalls noch Vicky Leandros' Schlagererfolg „Theo, wir fahr'n nach Lodz“ von 1974 freilich ohne in der Regel auch nur von der wesentlich älteren Geschichte des Liedes gehört zu haben (vgl. POLCUCH 1975; MeNSCHING 2006). Davon abgesehen existierte die Erinnerung an die Stadt lange fast nur noch in den Kreisen jener Deutschen, die aus der Stadt stammten und die bei Kriegsende 
Die Wiederentdeckung der polyethnischen Vergangenheit der Stadt Łódź

1944/1945/1946 freiwillig oder unfreiwillig die Stadt verlassen hatten. Die Stadt fand sich überwiegend in deren Erinnerungen, Memoiren und Publikationen aus dem Umfeld der Landsmannschaften der Vertriebenen wieder (EICHLER 1942; RENNER 1983; TREGER 1985; NASARSKI 1988; HEIKE 2002; RADZISZEWSKA 1999). Erst in jüngster Zeit taucht Lodz im Zuge der Wiederentdeckungen des immer noch weitgehend unbekannten östlichen Europas gelegentlich in Reisefeuilletons und -reportagen wieder auf (SCHLÖGEL 1996; RADA 2001; ZEH 2003; KROGERUS 2004; BRAUNS 2005; MENSCHING 2006). Wie bereits in den Reiseberichten und -reportagen des späten 19. und frühen 20. Jhd.s (DÖBLIN 1925; ROTH 1928; ERENBURG 1973) erscheint die Stadt den Betrachtern immer noch (oder bereits wieder?) als ein verwirrendes, faszinierendes, zugleich aber abstoßendes Kuriosum. Es gab und gibt also verschiedene Wahrnehmungen der Stadt, die zu entdecken sind, wenn man sich auf Lodz einlässt.

„Die Stadt als kultureller Raum“ (LINDNER 2004), ein Schlüsselbegriff der Moderne, wird zunehmend auch zu einem zentralen Erinnerungsort (CHÂTELLIER 2002). Sie steht damit nicht nur im Zentrum vielschichtiger kulturwissenschaftlicher Forschungsinteressen, sondern spielt auch in der nach dem Fall des ,Eisernen Vorhangs“ (Winston Churchill) in (Zentral-)Europa virulenten Diskussion um Regionalität, Identität und Vergangenheit eine wichtige Rolle. Während die Frage nach einer regionalen Identität teils auch deshalb schwer zu beantworten ist, weil Regionen im Sinne von Großräumen meist nur schwer abgrenzbar sind (JOACHIMSTHALER 2002, 2002a, 2002b), vereinfacht sich das Problem der Abgrenzung im Falle einer städtischen Regionalidentität etwas. Städte sind zumeist mehr oder minder klar umgrenzte Räume, die allerdings auf das Umland zurückwirken. Sie bieten sich als noch relativ überschaubar zur Untersuchung daher besonders an. Gerade zu zentraleuropäischen Orten, in denen auf Grund der Brüche des 20. Jhd.s die ursprüngliche, polyethnische Bevölkerung nicht mehr zu finden ist und es zum Teil nicht einmal mehr topographisch historische Kontinuitäten gibt, liegen inzwischen eine größere Zahl meist beeindruckende neuere Studien vor, etwa zu Breslau (BUŚKO / Goliński / KARZMAREK / ZiąTKOWSKI / KulaK / Suleja 2001; DAVIES / MoOrhouse 2002; VAN RAHDEN 2000; THum 2003), Danzig (LOEW 2003), Lublin (GEBHARD 2006), Riga (VON HIRSCHHAUSEN 2006), Budweis (KING 2002), Triest (MAGRIS / ARA 2005; LUNZER 2002) oder Czernowitz (BRAUN 2005; CORBEA-HOISIE 2003; HEPPNER 2000). Dabei fällt auf, dass viele dieser Untersuchungen nicht von Autor/inn/en aus den Städten selbst stammen. Vor Ort selbst gingen die Impulse für eine Hinwendung zur verdrängten oder vergessenen Vergangenheit meist von Fremden oder ,Außenseitern ' und nicht, 
wie auf den ersten Blick zu erwarten wäre, von der lokalen historischen Forschung aus. Dieser gelang es erst langsam, sich von den über Jahrzehnte gefestigten ideologischen Sichtweisen zu lösen.

Gerade in Polen war es dabei häufig die Germanistik, die sich daran machte, „deutsch-polnische Erfahrungswelten im Spannungsfeld von Fiktion und Geschichte“ (JOACHIMSTHALER 2002:11) zu rekonstruieren (JOACHIMSTHALER 2007). Vor allem in den Grenzregionen, den ehemals multiethnischen Gebieten, gab es für die junge Generation eine fremde unbekannte Vergangenheit (wieder) zu entdecken. Auch darüber hinaus - je mehr das vom Westen zunehmend vergessene Mitteleuropa wieder in den Blick geriet - wurden die regionale Geschichte und hier vor allem jüdische und deutsche Spuren ein Thema (BYCZKIEWICZ 2007; BoOCKMANN / BuCHHOLZ / CONZE 2002). Da die multikulturelle Vergangenheit eines Ortes, die nur begrenzt in der architektonischen Hinterlassenschaft erkennbar ist, vor allem in Erinnerungen, Literatur und u. U. in regionalen (sprachlichen) Besonderheiten lebendig und damit erfahrbar geblieben ist, bot sich die Suche nach diesen Spuren für die Germanistik geradezu an. Natürlich konnte eine verstärkte Auseinandersetzung mit dem Thema in Polen erst nach dem Ende der Volksrepublik und dem damit verbundenen Wegfall der ideologischen Vorgaben erfolgen - trotz einiger vorheriger vorsichtiger Versuche - wie JÜRGEN JOACHIMSTHALER (2007:27) in Erinnerung ruft:

Erhebliche Bedeutung gewann die in diesem Zusammenhang von Krzysztof A. Kuczyński gleich 1989 veranstaltete Konferenz zur „Literatur des Grenzlandes“, mit der einerseits eine erste Zusammenschau der verschiedenen regionalen Aktivitäten geleistet werden konnte und andererseits der Begriff an strategisch bedeutsamer Stelle in die Diskussion (wieder)eingeführt wurde, der die Diskussion in den folgenden Jahren beherrschen sollte: „Grenzland“. Unter „Grenzlandliteratur“ und „Grenzlandkultur“, unter „Grenzlandphänomenen“ jeglicher Art wurden hier insbesondere jene Interferenzerscheinungen verstanden, die auf sprachlicher, motivlicher, kultureller und emotionaler Ebene zu einer Vermischung polnischer und deutscher (und jüdischer und litauischer und vieler weiterer) Kulturelemente innerhalb eines Individuums (im literaturwissenschaftlichen Idealfall also eines Romanhelden oder eines lyrischen Ich oder eines Autors) oder Kollektivs führten oder diese widerspiegelten.

Damit hatte die Konferenz, die sozusagen als Initialzündung für die Auseinandersetzung mit dem Thema fungierte, zwar in Łódź stattgefunden (KUCZYŃSKI / SCHNEIDER 1991), letztlich sollte dies aber ohne größere Auswirkungen für die Auseinandersetzung mit der Stadtgeschichte und der regionalen Literatur zu Lodz selbst bleiben. Zwar beschäftigte sich Kuczyński seither immer wie- 
der auch mit einzelnen Lodzer Deutschen und ihren Werken, doch sein Interessengebiet blieben vorrangig die deutsch-polnischen Literaturbeziehungen in den Grenzregionen Polens, insbesondere Schlesiens. Zudem blieben seine Artikel oft faktografisch und häufig apologetisch. ${ }^{3}$ „Unkritische Apologien des deutschen Anteils der Vergangenheit der Stadt Lodz und Mittelpolens nutzen dagegen wenig", merkt WOLFGANG KESSLER (2002:164) zu Recht kritisch dazu an. Auch wenn KUCZYŃSKI (1999, KUCZYŃSKI / KUCHARSKA 1998; KUCZYŃSKI / BARTOSZEWSKA 2000) im Laufe der letzten Jahre immer wieder Konferenzen veranstaltete, auf denen auch die Lodzer Literatur Thema war, stießen diese Veranstaltungen kaum auf eine über eine punktuelle regionale Aufmerksamkeit hinausgehende Resonanz. Die fehlende Wahrnehmung der Stadt und ihrer Regionalgeschichte und -literatur, selbst innerhalb der Region und erst recht über diese hinaus, hängt zu einem guten Teil mit der erwähnten Unsichtbarkeit zusammen. Kurioserweise war es gerade jenes auf der 1989er Łódźer Konferenz in die Diskussion wieder eingebrachte Konzept des „Grenzlandes“ und der „Grenzlandliteratur“, das zu dieser erneuten Unsichtbarkeit - zumindest aus meiner Sicht - beigetragen hat. Dadurch nämlich fokussierte sich das Forschungsinteresse auf die ehemals deutschen Gebiete im Norden, Nordwesten und Westen sowie auf das ehemals österreichische Galizien im Südosten des heutigen Polen. In diesen ehemaligen und gegenwärtigen Grenzregionen waren Multikulturalität und Polyethnizität der Regelfall, Mehrsprachigkeit und gegebenenfalls eine situativ wechselnde Identität die Regel. Dort waren Vielvölkerstädte normal - insoweit ist Jacek Indelak zuzustimmen -, in zentralpolnischen Gebieten galt dies aber meist nur bezogen auf das Neben- und Miteinander von Polen und Juden. Multikulturalität und Polyethnizität in Lodz stellten insoweit eine Besonderheit dar, da sie weder geografisch bedingt noch historisch gewachsen waren. Sie sind vielmehr durch eine staatlich gesteuerte Ansiedlungspolitik erst bewusst geschaffen worden. Vor 1820 war Łódź eine fast rein polnische Kleinstadt - sieht man einmal von dem damals am Ort lebenden Dutzend Juden ab. Erst ab 1823 kamen Deutsche als Fachkräfte zum Aufbau einer Textilindustrie nach Lodz, vor allem aus dem Rheinland, der Eifel, Sachsen, Schlesien, Böhmen und anderen von den wirt-

Zwar wandte sich im Anschluss an die erste Konferenz mit Barbara Ratecka eine Mitarbeiterin an Kuczyńskis Lehrstuhl für Literatur und Kultur Deutschlands, Österreichs und der Schweiz dem Thema zu und behielt es auch nach seinem/ ihrem Wechsel 2001 an das von Kuczyński gegründete Institut für Deutschlandstudien bei, doch steht die mehrfach angekündigte Monographie zur deutschsprachigen Literatur und über Lodz immer noch aus. 
schaftlichen Folgen der Napoleonischen Kriege gebeutelten deutschen Textilindustriegebieten. Erst in den 1850er und 1860er Jahren stieg auch die Zahl der jüdischen und der polnischen Stadtbewohner beträchtlich an. Diese kamen nach dem Wegfall der Zollgrenzen, der teilweisen Emanzipation der Juden 1862 und der Abschaffung der Leibeigenschaft 1864 als Industriearbeiter nach Lodz. Nach Emanzipation und Bauernbefreiung verarmte die ländliche und kleinstädtische Bevölkerung immer mehr. Da diese Menschen aber nun das begrenzte Recht auf Freizügigkeit hatten, strömten viele von ihnen auf der Suche nach Arbeit in die Stadt. Nach der Überwindung der durch den gescheiterten polnischen Aufstand von 1863 und den nordamerikanischen Sezessionskrieg ausgelösten schweren Krisen nahm die Stadt schließlich ihren sagenhaften Aufschwung. Damit aber war innerhalb weniger Jahre eine ethnische Vielfalt in der Stadt entstanden, die es außerhalb der Lodzer Umgebung so in den unter russischer Verwaltung stehenden zentralpolnischen Gebieten nicht gab, abgesehen von der Hauptstadt Warschau vielleicht. Lodz war - wie es KARL SCHLÖGEL (1996:127) einmal treffend formulierte - eine „Grenzstadt - mitten in Polen“. Diese besondere Lage und historische Situation führten auch dazu, dass Lodz im Zusammenhang mit „Grenzlandkultur“, „Grenzlandliteratur" und „Grenzlandphänomenen" meist übersehen wird. So findet Lodz beispielsweise in JENS STÜBENs (2002:69f.) vielversprechendem Konzert einer „Geschichte der deutsch(sprachig)en Literatur im östlichen Europa“ keine Berücksichtigung - es sei denn, man möchte Lodz mit unter der Region ,Posen (Großpolen) ${ }^{6}$ subsumieren. ${ }^{4}$ Lodz fristet also auch in Bezug auf seine Wahrnehmung als multikulturelle mitteleuropäische Großstadt ein Schattendasein.

Selbst vor Ort sollte es relativ lange dauern, bis die Rückbesinnung auf diesen Teil der Lodzer Vergangenheit in größerem Umfang einsetzte. Erst Mitte der 1990er Jahre sollte sich die Situation langsam ändern. Nun begann sich auch auf Seiten der Lokalhistoriker die Einstellung zögerlich zu wandeln. Allerdings sollte es noch länger dauern, bis alte ideologische Scheuklappen weitgehend abgelegt wurden. Auch zur Lodzer Stadtgeschichte hatte sich die polnische Forschung - wenig verwunderlich - nach 1945 lange Zeit vor allem

4 Dies aber erscheint mir aus zwei Gründen nicht ratsam: Erstens lässt sich die historische Entwicklung von Lodz und Umgebung kaum mit der Posens vergleichen - ganz abgesehen von einer daraus resultierenden, völlig anders gearteten regionalen Identität; zweitens waren es die Nationalsozialisten, die während der Okkupation Polens 1939-1944 Lodz und Posen schon einmal in einem Territorium, dem sogenannten, Wartheland', zusammengefasst hatten. 
Die Wiederentdeckung der polyethnischen Vergangenheit der Stadt Łódź

ideologiekonform marxistisch mit der überwiegend polnischen Arbeiterschaft der Stadt befasst und die Polyethnizität von Lodz weitgehend ignoriert bzw. mehr oder minder verzerrt dargestellt (GINSBERT 1962; ROSSET 1929; ROSIN / BARANOWSKI / FIJAEEK 1980; BARSZCZEWSKA-KRUPA / SAMUŚ / WACHOWSKA / CZAPLIŃSKI / NAZAREWICZ / BANASIAK 1985). Erleichtert wurde dies zudem dadurch, dass durch die Ausblendung der deutschen und jüdischen Handwerker- bzw. Arbeiterschaft, der (marxistische) Klassengegensatz so auch zum nationalen Antagonismus wurde: polnische Arbeiter auf der einen, deutsche und jüdische Fabrikanten, d.h. Kapitalisten, auf der anderen Seite. Damit war dem nationalen polnischen Selbstbild gedient wie auch der verordneten Staatsideologie. Allerdings findet sich schon in der Zeit zwischen den Kriegen keine eigenständige Monographie eines polnischen Historikers, die die Multikulturalität der Stadt thematisiert, da die Stadt bereits damals weitgehend außerhalb des polnischen Wahrnehmungshorizontes lag. Größere Arbeiten zu Lodz stammen meist von deutschen (FLATT 18535; KOSSMANN 1966 ${ }^{6}$ ) oder jüdischen (ALPERIN 1928; FRIEDMAN 1935; HERTZ 1933; BANNER 1938) Autoren - wenn auch nicht ausschließlich (ROSSET 1929). Nach 1990 war die Entwicklung ähnlich. Auch hier kamen die Impulse meist von außen, und das im doppelten Sinne: zum einen thematisch vor allem durch die Auseinandersetzung mit der jüdischen Geschichte auch vor 1939; zum anderen, weil die Anregungen zu dieser Auseinandersetzung nicht von Lodzer Historikern selbst stammten, sondern Außenseiter sich des Themas annahmen. So wird bereits 1991 ein erster Sammelband zur Geschichte der Lodzer Juden veröffentlicht. Dieser geht aber auf eine Kooperation mit dem Institute for Polish-Jewish Studies in Oxford zurück (Polonsky 1991; PUŚ / LISZEWSKI 1991). Die erste Tagung zum multikulturellen Lodz ging auf einen 1993 gemachten Vorschlag des damaligen Direktors des Deutschen Historischen Instituts Warschau, Rex Rexheuser, zurück. Sie fand schließlich als internationale, interdisziplinäre Konferenz „Gruppenbeziehungen in einer multiethnischen Stadtgesellschaft: Polen, Deutsche und Juden in Lodz im 19. und 20. Jahrhundert (bis 1939)“ vom 19.-22. Oktober 1995 in Łódź statt (SAMUŚ 1997; Hensel 1999). Das öffentliche Echo war zwar noch gering, dennoch setzte zumindest in akademischen Kreisen ein Umdenken ein. STEFAN PYTLAS (1994) war kurz zuvor der

5 Oskar Flatt, der Autor der ersten Monographie zu Lodz überhaupt, war, auch wenn das Buch 1853 in Warschau auf Polnisch erschien, anscheinend deutscher Herkunft.

6 Oskar Kossmanns Studie geht laut Aussagen des Autors auf seine verschollene Krakauer Dissertation von 1932 zurück (KossmanN 1966:XIV). 
erste Historiker gewesen, der sich explizit von einem auf das Polnische und die Arbeiterschaft zentrierten historiographischen Lodzbild ab- und den Lodzer Industriellen zuwandte. Zugleich richtete er damit den Blick auf die nicht-polnischen Ethnien - freilich ohne sich gänzlich von der tradierten Vorstellung vom skrupellos ausbeutenden, für seinen Profit zu allem bereiten Fabrikanten lösen zu können. BIANKA PIETROW-ENNKER (2005:171, Anm. 9) merkt nicht ganz zu unrecht kritisch an, er bediene weiter , ,...] trotz höchst verdienstvoller Untersuchungen [...] implizit das negative Stereotyp des Unternehmers: eines egoistischen Typus' mit unterstellter krimineller Energie, systematisch zu Unehrlichkeit und Betrug bereit. Daher ist für Pytlas die Redlichkeit ein zentraler Maßstab seiner Bewertung." Trotz dieser Kritik kann die Studie als richtungweisend gelten, wenigstens bezogen auf die weitere historische Forschung zu Lodz im 19. Jhd. Neben der Germanistik und der Geschichtswissenschaft gingen weitere Impulse von der Theaterwissenschaft aus. ANNA KULIGOWSKAKORZENIEWSKA $(1976,1995,1997,1999)$ begann mit Untersuchungen zum polnischen Lodzer Theater und eröffnete damit ein Forschungsfeld, das sich bald auch auf die übrige Lodzer Theaterlandschaft erstrecken sollte. Vor allem die von ihr betreute, ebenfalls 1994 vorgelegte Magisterarbeit des Kulturwissenschaftlers und Germanisten ARTUR PEŁKA (1994, vgl. auch 2005) hat Pioniercharakter, da mit ihr die Beschäftigung mit dem Deutschen Theater einsetzte, die inzwischen zur Publikation einer eigenständigen Monographie durch Karolina PRYKowsKa-MichalaK (2005) zu diesem Thema geführt hat. Auch zum jüdischen Theater setzte seitdem eine rege Forschungstätigkeit ein (LEYKO 1992; KULIGOWSKA-KORZENIEWSKA / LEYKO 1998; LEYKO 2000). Zumindest in akademischen Kreisen war die multikulturelle Lodzer Vergangenheit ab Mitte der 1990er Jahre zu einem Thema geworden.

Zur selben Zeit begann auch auf anderer Ebene sich das Interesse an der Vergangenheit von Lodz zu entwickeln. Krystyna Radziszewska begab sich mit Germanistikstudentinnen und -studenten ganz konkret auf Spurensuche nach Relikten der deutschen Vergangenheit im Lodzer Stadtbild. Die Anregung hierfür hatte sie nicht in Łódź selbst erhalten, sondern durch ein Projekt von SCHMIDT / DEWERTMANN / RUSAK (1993) mit Danziger Studentinnen und Studenten, die einen Stadtführer durch Gdańsk auf den Spuren von Günter Grass und seinen Danziger Romanen verfasst hatten. Allerdings ließ sich für einen Lodzer Stadtführer auf deutschen Spuren nur schwer ein Verlag finden. Lange war niemand bereit, das Wagnis einzugehen, einen bilingualen - deutsch-polnischen - Stadtführer herauszubringen, der sich explizit mit der deutschen Vergangenheit befasst. 1997 schließlich konnte das Buch (RADZISZEWSKA 1997), das seitdem mehrere Auflagen erlebt hat, endlich erscheinen. Zu diesem Zeit- 
Die Wiederentdeckung der polyethnischen Vergangenheit der Stadt Łódź

punkt war das Nachfolgeprojekt RADZISZEWSKAs (1999) bereits relativ weit fortgeschritten. War es bei dem ersten Buch noch um das Auffinden der Spuren am Ort und um die Darstellung dieser Erinnerungsorte gegangen, so beschäftigten sich die Studentinnen und Studenten nun mit jenen Deutschen, die bis zum Ende des Zweiten Weltkriegs in Lodz gelebt hatten und mit deren (Kindheits-)Erinnerungen. Zu diesem Zweck hatten sie auf einem landsmannschaftlichen Treffen in Stuttgart deutsche Lodzer und Lodzerinnen interviewt und waren nun dabei, die Interviews redaktionell zu bearbeiten und für die Veröffentlichung vorzubereiten. In der Zwischenzeit hatte sich auch sonst innerhalb des wissenschaftlichen universitären Betriebs einiges getan: Der von MARIAN WILK (1996) herausgegebene polnische Konferenzband zu den Lodzer Deutschen vor 1939 bot Interessierten einen ersten Überblick über die regionale deutsche Geschichte und Literatur, auch wenn ein Teil der unter anderen von Germanist/inn/en verfassten Artikel weitgehend deskriptiv blieben. Im November 1997 veranstaltete Krzysztof A. Kuczyński (KUCZYŃSKI / KUCHARSKA 1998) eine Konferenz zu Ehren der aus Lodz stammenden Schriftstellerin und Übersetzerin Gerda Leber-Hagenau. Auch die Teilnehmerinnen und Teilnehmer der Konferenz zur Stadtgeschichte zwei Jahre vorher trafen sich anlässlich der Veröffentlichung des ersten Tagungsbandes (SAMUŚ 1997) erneut in Łódź zu einer eintägigen Tagung, um weitere Forschungsdesiderata zu diskutieren. Dabei wurde auch konstatiert, dass sich die Situation in Bezug auf das Interesse der Öffentlichkeit vor Ort verbessert habe. Mit der verstärkten Aufmerksamkeit ging aber eine zunehmende und weiterhin andauernde Verklärung der multikulturellen Vergangenheit der Stadt einher. Dies veranlasste JACEK INDELAK (1997:2718) bereits damals - 1997 - in seinem für die Lodz gewidmete Ausgabe der in Deutschland erscheinenden Zeitschrift StadtBauwelt, der Vierteljahreshefte der Bauwelt verfassten Artikel zu der nicht unberechtigten polemischen Bemerkung: „Noch ein wenig und Lodz wird zur Wiege der Ökumene ausgerufen. Ach was, ach was, zur Vorkämpferin des vereinigten Europas..." Diese für Außenstehende wahrscheinlich überraschende Entwicklung ist auch vor dem Hintergrund eines für Lodz typischen historischen Phänomens zu sehen und wird durch dessen Wiederentdeckung und -belebung im Laufe der letzten zehn Jahre verständlicher: Die einzelnen ethnischen Gruppen hatten zwar im 19. und frühen 20. Jhd. also mehr nebenals miteinander gelebt, zugleich aber entwickelten sie ein neues lokales Selbstverständnis. Die Mehrheit sah sich unabhängig von der ethnischen und nationalen Herkunft und Zugehörigkeit (auch oder vor allem) als Lodzer. Es entstand das Bild vom ,Lodzermenschen“. Ein ,Lodzermensch`war jemand, der in der neuen Zeit und der Moderne mit ihrer Industrialisierung eine Chance auf 
ein Auskommen, wenn nicht gar auf die große ,amerikanische' Karriere vom Tellerwäscher zum Millionär sah. Dabei war es letztlich für die Menschen, die in dieser Stadt lebten, für die Erfüllung ihrer Träume nebensächlich, ob sie nun Polen, Juden oder Deutsche waren. Die Karrieren von Ludwig (Luis) Gayer, Carl Scheibler, Julius Heinzel, Julius Kunitzer, Traugott Grohmann oder Robert Biedermann, die als deutsche Webermeister, Ingenieure und Facharbeiter in die Stadt gekommen waren und es teils zu immensem Reichtum gebracht hatten, zeigten, welche Chancen sich in der Stadt boten. Darüber hinaus hatten es zudem auch einige aus der Masse der jüdischen Zuwanderer an die Spitze geschafft: Oft hatten sie als Kleinhändler, Kommissionäre oder Verwalter begonnen und waren (Multi)millionäre geworden, wie Israel Poznański - der nach Carl Scheibler zweitreichste Lodzer -, aber unter anderen auch Markus Silberstein, Abraham Prussak, Szaja Rosenblatt, die Brüder Jarociński oder Oszer Kon. Auch wenn die Mehrheit der Lodzer Bevölkerung meist kaum genug zum Leben hatte, stand ihnen die Möglichkeit der großen Karriere immer lebhaft vor Augen. Während man außerhalb von Lodz unter einem ,Lodzermenschen " vor allem einen rücksichtslosen Karrieristen sah, der Herkunft und Tradition zu Gunsten der Aussicht auf Reichtum aufgegeben hatte, war es in Lodz selbst erstrebenswert, ein echter ,Lodzermensch' zu sein. Zur heutigen Bedeutung dieses historischen Phänomens bemerkte WOLFGANG KeSSLER (2002:163f): „Der ,Lodzermensch', zeitgenössisch eher ein Vorwurf nationaler Indifferenz, hat auf dem Weg Polens in die Europäische Union als Muster vorbildlicher Interkulturalität eine erstaunliche Wiederbelebung erfahren." Jacek Indelaks Aussage sollte sich also als geradezu prophetisch erweisen, was auf der kleinen Historikertagung 1997 bestenfalls zu ahnen war (BUDZIAREK / SALM / WeSOŁOWSKI 2003; MACHEJEK 2004, 2005; KoteR / KULESZA / PuŚ / PYTLAS 2005).

Etwas anderes wurde mir aber dort damals deutlich, dass nämlich die Historiker sich nicht wirklich darüber im Klaren waren, was in der Germanistik geschah und umgekehrt. Die studentischen Projekte wurden teils belächelt, teils als ungebührliche Konkurrenz angesehen, da die Initiative von Teilen der Germanistik ausging. Generell war man allerdings auf beiden Seiten einer Kooperation nicht abgeneigt, unterstellte allerdings, die jeweils andere Seite sei nicht interessiert. Nachdem diese Missverständnisse ausgeräumt werden konnten, kam es beim nächsten stadtgeschichtlichen Projekt schließlich zu einer Zusammenarbeit zwischen Germanist/inn/en und Historiker/inne/n. Diesmal ging es um das Mit- und Nebeneinander von Polen, Juden und Deutschen vor 1939, wie es sich vor allem in der mehrsprachigen Lokalpresse und literarischen Texten widerspiegelt (RADZISZEWSKA / WOŹNIAK 2000). Zwar gab es 
auch weiterhin gelegentlich Vorbehalte gegen die Projekte, u. a. von Teilen der jüdischen Gemeinde und der kleinen deutschen Minderheit in Łódź, doch stieß das Thema auf ein immer größeres Interesse in der Öffentlichkeit. Der Autor und Filmemacher Leszek Skrzydło produzierte 1994-1997 für das erste Programm des Polnischen Fernsehens (TVP 1) eine Dokumentarfilmserie über die wichtigsten, legendären deutschen und jüdischen Fabrikantenfamilien (SKRZYDŁO 1999, 2000, 2007). Mehrere Verlage nahmen sich nun auch außerhalb der Universität des Themas Lodz an - zumeist allerdings auf Initiative von Einzelpersonen. In der Folgezeit erschienen etliche auch für ein breiteres Publikum gedachte Werke zur multikulturellen Stadtgeschichte, darunter auch einige biographische Studien zu bekannteren deutschen Fabrikantenfamilien (BARTCZAK 1999; KOWALSKI 1999; KUŹKO 2000; BUDZIAREK 2000; STEINHAGEN 2003). Daneben gab es auch kurze Überblicksdarstellungen, die, sich auf ausführlichere Untersuchungen stützend (SKRZYDŁO 2000; BUDZIAREK 2003; SZUKALAK 1990), vor allem als touristische Information gedacht waren und deshalb entweder zweisprachig (BUDZIAREK 2000; SKRZYDLO 2000; SZUKALAK 1990) oder in je einer polnischen, deutschen und englischen Ausgabe veröffentlicht wurden (PODOLSKA 2004, 2005, 2006). Die zu den Lodzer Juden vor 1914 bereits 1998 publizierte erste Monographie (Puś 1998), die zu einem verstärkten Interresse an der Geschichte der Lodzer Juden beigetragen hatte (SPODENKIEWICZ 1998, 2007; WALICKI 2000; BADZIAK / WALICKI 2002; KEMPA / SZUKALAK 2001-2004; SZUKALAK 2007), erschien 2001 in der zweiten Auflage. Im selben Jahr brachte das Institut für Deutschlandstudien auch einen Sammelband zu den Deutschen in Lodz vor 1945 heraus (KUCZYŃSKI / RATECKA 2001), in dem versucht wurde, ein Gesamtbild zu liefern. Einige Beiträge blieben dabei allerdings hinter dem bis dahin erreichten Forschungsstand leider zurück, während andere Einblicke in neue Forschungsfelder sowohl der Geschichts-, Literatur-, Kultur - und Sprachwissenschaft lieferten. Als der vierte Band aus der Reihe der von KRYSTYNA RADZISZEWSKA (2002) herausgegebenen und von Studenten und Studentinnen verfassten zweisprachigen Bücher erschien - diesmal zu den Jahren 1939-1944 - waren die Regionalstadtgeschichte und -literatur innerhalb und außerhalb der Germanistik weitgehend als Thema etabliert. Im Laufe der nächsten Jahre entstanden etliche Magisterarbeiten sowohl im Rahmen der Landeskunde und Kulturwissenschaft als auch der Sprachwissenschaft, die sich meistens auf der Grundlage der deutschsprachigen Presse der zweiten Hälfte des 19. und der ersten Hälfte des 20. Jhd.s mit verschiedensten regionalen Spezifika be- 
fassten. ${ }^{7}$ Auch an der Łódźer Ethnologie (KOPCZYŃSKA-JAwORSKA 1999) und der Polonistik beschäftigten sich einzelne, hier vor allem MARIA KAMIŃSKA (1997, 1999, s. nun auch BIEŃKOWSKA / CYBULSKI / UMIŃSKA-TYTON 2007) seit längerem mit Regionalismen und dialektalen bzw. soziolektischen, aus dem Deutschen oder Jiddischen herrührenden Einflüssen auf das Polnisch der Region. Innerhalb der germanistischen Sprachwissenschaft beginnt man das Ganze aus dem entgegengesetzten Blickwinkel zu betrachten (unter anderem BRITT-MARIE SCHUSTER 2005; MICHOŃ 2005; WEIGT 2005; SADZIŃSKI 2005), freilich ohne auf eine so umfangreiche Feldforschung und langjährige Erfahrung zurückgreifen zu können wie in der Polonistik oder der Ethnologie. Aber innerhalb der Germanistik entwickelt sich langsam eine Forschungstradition. Abgesehen von Krystyna Radziszewskas kontinuierlichen Forschungen wurde nämlich auch für einige der Studierenden die Beschäftigung mit Lodz sogar über Jahre hinweg zu einem eigenständigen Betätigungsfeld. Jene Student/inn/en, die im zweiten Studienjahr an dem Buch zur deutschen Besetzung von Lodz im Zweiten Weltkrieg und zum Lodzer Ghetto mitgearbeitet und Kapitel zu einzelnen Aspekten verfasst hatten, sollten später auch ihre Magisterarbeiten dazu schreiben.

Das Thema der deutschen Besetzung der Stadt im Zweiten Weltkrieg und das des von den Nationalsozialisten 1940 errichteten und bis 1944 existierenden jüdischen Ghettos sollte zu einem der Schwerpunkte an der Germanistik im Rahmen der Auseinandersetzung mit der regionalen Vergangenheit werden. Entscheidend hierfür war vor allem die Kooperation im Rahmen einer vom DAAD geförderten Germanistischen Institutspartnerschaft mit der Universität Gießen und der Forschungsstelle für Holocaustliteratur. Im Rahmen dieser Zusammenarbeit wurden die sogenannte Ghetto Chronik (FEUCHERT / LEIBFRIED / RIECKE / BARANOWSKI 2004; FEUCHERT / LEIBFRIED / RIECKE 2007) sowie weitere Texte aus deren Umfeld ediert und auf Polnisch und Deutsch herausgegeben. (SINGER 2002; LÖW 2006) Eine Edition der sogenannten „Ghetto Enzyklopädie“ ist in Vorbereitung. Im Umfeld bzw. im Anschluss an die lokalen Gedenkveranstaltungen anlässlich des 60. Jahrestags der Ghettoauflösung erschienen 2004 einige Werke zum Ghetto und zur deutschen Besetzung der Stadt (FEUCHERT / LEIBFRIED / RIECKE / BARANOWSKI 2004; RADZISZEWSKA / RIECKE 2004) und zur jüdischen Stadtgeschichte (MACHEJEK 2004). Die bereits auf der kleinen Historikertagung von 1997 angeregte Folge-

7 Demnächst sind sogar die ersten sprachwissenschaftlichen wie kultur- und literaturwissenschaftlichen Dissertationen von Marcin Michoń und Anna Sawicka zu erwarten. 
tagung zur deutschen Okkupation der Stadt und zum Lodzer Ghetto fand nun endlich ebenfalls statt (SAMUŚ / Pú́ 2006). Durch diese dem Zeitpunkt und dem Zeitgeist geschuldete verstärkte Auseinandersetzung mit dem Ende der polyethnischen und multikulturellen Stadtgeschichte geriet diese selbst etwas aus dem Blick. Zwar wurde im Rahmen des deutsch-polnischen Jahres 20052006 ein zweisprachiger Band zur Geschichte der Deutschen in Lodz publiziert (MACHEJEK 2005), und der Lehrstuhl für Deutschlandstudien (Katedra Badań Niemcoznawczych) veranstaltete in Zusammenarbeit mit dem Historischen Institut und der Stadtverwaltung eine groß angekündigte Konferenz zu den Deutschen in Lodz (KUCZYŃSKI 2005), nur gingen die dort präsentierten Erkenntnisse kaum über die aus dem 2001 veröffentlichten Band hinaus (KUCZYŃSKI / RATECKA 2001). Eine deutschsprachige Anthologie mit Texten zu Lodz, die bereits Jahre zuvor in Kooperation mit der Germanistik der Universität Chemnitz begonnen worden war, erschien nun endlich ebenfalls (KUCZYŃSKI / RATECKA / MEHNERT 2005). Auch wenn die Textauswahl weitgehend repräsentativ und einleuchtend, das Projekt an sich sehr verdienstvoll ist, da bis dahin nur polnische Anthologien vorlagen (STOLARZEWCZ 1935; FREJDLICH 1973; SKIBIŃSKI / STELMASZCZYK-ŚWIONTEK 1982; WILMAŃSKI 1993; GÓRECKI 1998), so hat das im Łódźer Universitätsverlag publizierte Werk aus meiner Sicht den Nachteil, dass es nur auf Deutsch erschienen ist. Zudem ist das einleitende Vorwort der Herausgeber fehlerhaft und unkritisch. Es schreibt damit den sich zunehmend verselbstständigenden Mythos von der „Stadt der vier Kulturen“ (BudZIAREK / SALM / WESOŁOWSKI 2003), in der diese harmonisch miteinander lebten, unhinterfragt fort (KUCZYŃSKI / RATECKA 2005). Darüber hinaus ist die enthaltene Bibliographie geradezu fahrlässig ungenau. Auch um dieses Manko zu beheben, entsteht im Rahmen des Germanisten-Zirkels der Lodzer Germanistik nun eine zweisprachige Anthologie. Die Veröffentlichung weiterer, heutzutage weitgehend vergessener deutschsprachiger literarischer Werke zu Lodz (GEYER 2002) ist in Vorbereitung (TEPLITZKA 1913, 2008) oder in Planung. Die Auseinandersetzung am Ort mit der lokalen Geschichte, einschließlich der in der und über die Region entstandenen Literatur kann mit Fug und Recht als vielfältig bezeichnet werden. Zur germanistischen Literatur-, Kultur- und Sprachwissenschaft sowie der Ethnologie und der Kultur- und Geschichtswissenschaften kommen nun auch verstärkt Studien aus dem Bereich der Architektur- und der Kunstgeschichte hinzu (STEFAŃSKI 2003, 2005, 2006; JORDAN o. J. [2007]). Außerdem sollte man nicht vergessen, dass auch über die vor Ort geleistete Forschung hinaus die Lodzer Stadtgeschichte zunehmend zu einem Untersuchungsgegenstand wird. So erscheinen inzwischen auch außerhalb von Łódź 
zunehmend Untersuchungen, in denen die Stadt eine Rolle spielt (BRANDES 1996; GuESNET 1997, 1997a, 1998, 1999; HOFMANN 2002, 2002a, 2004, 2004a; PIETROW-ENNKER 1995, 1999, 2002, 2002a, 2003, 2005; KOSSERT 2006; F. M. SCHUSTER 2004, 2007, 2007a, 2007b sowie SCHUSTER / CAYSA 2006). Auch wenn man in den letzten zehn bis zwanzig Jahren in Łódź ebenfalls dabei ist, die polyethnische Vergangenheit der Stadt wieder zu entdecken - bis hin zur mythischen Verklärung im Zuge einer Ankurbelung des Tourismus in der Region - so bleibt doch aus meiner Sicht noch einiges zu tun. Beispielsweise ist bisher kaum erklärt worden, was Lodz von anderen Städten und Regionen mit multikultureller Bewohnerschaft unterscheidet, obwohl historisch bedingte Unterschiede beispielsweise zu Schlesien, Galizien oder Danzig kaum bestreitbar sind. Was über ethnische und religiöse Grenzen hinweg die Menschen in Lodz miteinander verband oder voneinander trennte, bleibt weiterhin ziemlich unklar - ganz abgesehen von dem Selbstverständnis des Einzelnen, seiner Selbstdefinition und Identität. Mit anderen Worten: Was zeichnet ihn aus, jenen ,Lodzermenschen', von dem gerade in Łódź selbst in den letzten Jahren wieder verstärkt die Rede ist? ? $^{8}$

Wie die schwierige Nachbarschaft (HENSEL 1999) konkret aussah und welchen äußeren Einflüssen das fragile Gleichgewicht am Ort ausgesetzt war, bedarf letztlich weiterer komparatistischer Untersuchungen. Als Grundlage dafür böten sich hier die in den verschiedensten Sprachen verfassten journalistischen und literarischen Texte aus und über Lodz an. Zwar wurde das Lodzer Pressewesen bereits systematisch erfasst (KASZUBINA 1967) und Beiträge der Lokalzeitungen bereits bei den unterschiedlichsten Fragestellungen mit herangezogen (KUCNER 2001, 2005; RADZISZEWSKA 2005), eine vergleichende, sich auf die Lokalpresse stützende Arbeit fehlt allerdings bisher. Auch die vielfältigen literarischen Texte über Lodz werden in letzter Zeit verstärkt gesammelt und teils erneut publiziert, so dass eine gute Basis vorliegt. Allerdings fehlen auch hier bisher vergleichende Studien, die sich mit den Bildern der Stadt in Belletristik und Erinnerungen kritisch auseinandersetzen und mit anderen historischen Quellen kontrastieren. Dies ist vor dem Hintergrund der bereits erwähnten, durch literarische Texte dominierten Wahrnehmung der Stadt umso spannender, weshalb ich selbst mich des Themas angenommen

8 Dieser Frage soll u. a. auf einer von der Kommission für die Geschichte der Deutschen in Polen e. V. veranstalteten und von Krystyna Radziszewska organisierten internationalen und interdisziplinären Konferenz im Oktober 2008 in Lodz nachgegangen werden. 
habe. ${ }^{9}$ So wichtig wie die Auseinandersetzung mit dem Ghetto und der Besetzung der Stadt durch die Deutschen ist, besteht doch die Gefahr, dass die Shoa die Wahrnehmung dominiert und alles, was davor lag, in den Schatten stellt, wie FRANÇOIS GUESNET (1998:13) feststellte: „In seiner Totalität scheint dieses Verbrechen nicht nur die Menschen, sondern auch ihre Geschichte vernichtet zu haben, indem es unsere Wahrnehmung auf sich zu fixieren sucht." Gerade für viele Opfer, die Zeugnis abgelegt haben, spielte dabei die Überlieferung des Lebens vor der Vernichtung eine mindestens genauso große Rolle wie die Darstellung des Lebens und Überlebens im Ghetto und in den Konzentrationslagern. Deshalb sind etliche Werke über das Lodzer Ghetto zugleich auch Texte über Lodz vor 1939 - was allerdings häufig übersehen oder nicht wahrgenommen wird. Damit steht der Forschung zur Lodzer Stadtgeschichte auch ein Textkorpus zur Verfügung, das bisher überwiegend nur im Rahmen der Auseinandersetzung mit der Holocaustliteratur zur Kenntnis genommen wurde. Die hier kurz dargestellte bisherige interdisziplinäre Forschung zu Lodz und die abschließend skizzierten möglichen Ansätze für weitere Untersuchungen machen eines deutlich: Die Lodzer Stadtgeschichte, die regionale Literatur und die Auseinandersetzung damit sind nicht weniger kontrovers und spannend als beispielsweise die Danzigs oder Breslaus. Lodz gilt zu Unrecht als unsichtbare, verborgene, von Geistern und Mythen heimgesuchte Stadt ohne Vergangenheit und Erinnerung.

\section{Literatur}

AksZTAJn, ARIE (1996): Ciotka Ester. [Tante Ester.] Übersetzt aus dem Hebräischen von Sara Bande-Celnik. Łódź.

Alberti, Michael (2006): Die Verfolgung und Vernichtung der Juden im Reichsgau Wartheland 1939-1945. Wiesbaden.

AlPerin, AAron (1928): Żydzi w Lodzi: Poczatki gminy Żydowskiej, 1780-1822. [Juden in Lodz. Die Anfänge der jüdischen Gemeinde in Lodz, 1780-1822.] Łódź.

[ANONYM] (2002) [=Jürgen Joachimsthaler, Aleksandra Bochenek, Jarosław Bogacki, Ulrike Fügl, Gabriella Jelitto, Grzegorz Jureczko, Maria K. Lasatowicz, Sebastian Mrożek, Daniela Pełka, Michael Rohrwasser, Andrea Rudolph, Izabela Surynt, Magdalena Sutarzewicz, Wiesław Śliwak, Monika Witt, Marek Zybura und Janusz Zydroń] (eds.): Regionalität als Kategorie der Sprach- und Literaturwissenschaft. Hrsg. vom Instytut Filologii Germańskiej der Uniwersytet Opolski. Frankfurt (M.)/Berlin/ Bern u. a.

9 Als theoretische Basis stehen inzwischen einige kulturwissenschaftliche Ansätze zur Verfügung ([Anonym] 2002; JOACHIMSTHALER 2007; ERLL 2003, 2005, 2005a; NEUMANN 2005, 2005a). 
BADZIAK, KAZIMIERZ / WALICKI, JACEK (2002): Żydowskie organizacje społeczne $w$ Łodzi (do 1939 r). [Jüdische Wohltätigkeitsorganistaionen in Lodz (bis 1939).] Łódź.

BANNER, HERSz (1938): Gmina żydowska w Łodzi. Krótki zarys dziejów ustrojowogospodarczych. [Die jüdische Gemeinde in Lodz. Kurzer Abriss der Organistions- und Wirtschaftsgeschichte]. Łódź.

BarszczewsKa-Krupa, Alina / Samuś, PaweŁ / Wachowska, Barbara / CzapliŃSKI, TADEUSZ / NAZAREWICZ, RYSZARD / BANASIAK, STEFAN (1985): W dymach czarnych budzi się Łódź. Z dziejów tódzkiego ruchu robotniczego 1882-1948. [Im schwarzen Rauch erwacht Lodz. Zur Geschichte der Lodzer Arbeiterbewegung 1882-1948.] Łódź.

BARTCZAK, MirosŁaW (1999): Scheiblerowie. [Die Scheiblers]. Łódź.

BARTKIEWICZ, Zygmunt (1911): Zte miasto. Obrazy z r. 1907. [Die böse Stadt. Bilder aus dem Jahr 1907]. Warszawa. Neu hrsg. unter dem Titel Złe miasto. [Die böse Stadt.] Łódź.

Bieńkowska, Danuta / Cybulski, Marek / Umińska-Tyton, ElżBieta (2007): Stownik dwudziestowiecznej Łodzi. Konteksty historyczne, spoteczne, kulturowe. [Handbuch zu Lodz im 20. Jahrhundert. Historischer, gegenwärtiger und kultureller Kontext]. Łódź.

Bloch, Karola (1981): Aus meinem Leben. Pfullingen.

Boockmann, Hartmut / Buchholz, Werner / Conze, Werner (ed.) (2002): Deutsche Geschichte im Osten Europas. 10 Bde. Berlin.

BRANDES, HARALD (1996): Historische Stadtstrukturen von Łódź unter dem Aspekt ethnisch/kultureller Vielfalt. In: MAIER, ROBERT / STÖBER, GEORG (eds.): Zwischen Abgrenzung und Assimilation - Deutsche, Polen, Juden. Schauplätze ihres Zusammenlebens von der Zeit der Aufklärung bis zum Beginn des Zweiten Weltkrieges. Hannover, 175-184.

Braun, Helmut (ed.) (2005): Czernowitz: Die Geschichte einer untergegangenen Kulturmetropole. Berlin.

BRAUnS, DiRK (2005): Die Schuhputzer der Katarzyna S. In: Berliner Zeitung, 26.11.2005:M01.

BudziAREK, MAREK (2000): Łodzianie. [Lodzer]. Łódź.

- (2003): Łódź Lodsch Litzmannstadt. Wyncinki z życia mieszkańców okupowanego miasta. [Łódź Lodsch Litzmannstadt. Ausschnitte aus dem Leben der Einwohner einer besetzten Stadt.] Łódź.

Budziarek, Marek / Salm, Jan / WeSOŁOWSKi, JaCEK (2003): Łódź, Lodz, Лod3b, לודז [sic]. Miasto czterech kultur. [Stadt der vier Kulturen]. Łódź.

BudziareK, Marek / Skrzydlo, Leszek / Szukalak, Marek (2000): Łódź nasze miasto. Unsere Stadt Lodz. Übersetzt von Małgorzata Jaroniek. Łódź.

Buśko, CeZARy / Goliński, Mateusz / KARZMAReK, Micha€ / ZiĄTKowsKi, LesZeK / KulaK, Teresa / Suleja, WŁodzimierz (2001): Historia Wroctawia. [Die Geschichte Breslaus]. 3 Bde. Wrocław. 
Die Wiederentdeckung der polyethnischen Vergangenheit der Stadt Łódź

ByCZKIEWICZ ANNA (2007): Die neueste deutschsprachige Reiseliteratur zu Galizien. In: SCHMitZ, WALteR / JoACHIMSTHALER, JÜRGEN (eds.): Zwischeneuropa - Mitteleuropa. Sprache und Literatur in interkultureller Konstellation. Akten des Gründungskongresses des Mitteldeutschen Germanistenverbandes. Hrsg. im Auftrag des Mitteleuropäischen Germanistenverbandes. Dresden, 419-429.

Calvino, Italo (2007): Die unsichtbaren Städte. Übersetzt von Burkhard Körber. München.

ChÂtellier, Hildegard ( $\left.{ }^{2} 2002\right)$ : Molloch Großstadt. In: FrançOIS, Etienne / SCHUlze, HAGEN (eds.): Deutsche Erinnerungsorte. Bd. 2. München, 567-583.

CORBEA-HoISIE, ANDREI (2003): Czernowitzer Geschichten. Über eine städtische Kultur in Mittel(Ost)-Europa. Wien/Köln/Weimar.

Davies, Norman / Moorhouse, Roger (2002): Microcosm. A Portrait of a Central European City. London.

- (2002): Breslau - Die Blume Europas. Die Geschichte einer mitteleuropäischen Stadt. Übersetzt von Thomas Bertram. München.

Döblin, Alfred (1925): Reise in Polen. Berlin/Frankfurt (M.).

Eichler, Adolf (1942): Deutschtum im Schatten des Ostens. Dresden.

ERENBURG, ILIA ( ${ }^{1} 1928$ / 1973): Łodź biega nago po świecie. [Lodz läuft nackt durch die Welt.] In: FREJDLICH, 87-94.

ERLL, ASTRID (2003): Gedächtnisromane. Literatur über den Ersten Weltkrieg als Medium englischer und deutscher Erinnerungskulturen in den 1920er Jahren. Trier.

- (2005): Literatur als Medium des kollektiven Gedächtnisses. In: ERLL / NüNNING, 248-276.

- (2005a): Kollektives Gedächtnis und Erinnerungskulturen. Eine Einführung. Stuttgart/Weimar.

ERLL, ASTRID / NÜNNING, ANSGAR (eds.) (2005): Gedächtniskonzepte der Literaturwissenschaft. Theoretische Grundlagen und Anwendungsperspektiven. Berlin/New York.

Feuchert, SASCha / Leibfried, ERWin / Riecke, JÖRg / BARANOWSKi, Julian / RAdZISZEWSKa, Krystyna (eds.) (2004): Letzte Tage. Die Lodzer Getto-Chronik. Juni/Juli 1944. Göttingen (=Schriftenreihe zur Lodzer Getto-Chronik).

FEUChERT, SASCHA / LeIBFrIEd ERwIN / RIECKE, JÖRG (eds.) (2007): Die Chronik des Gettos Lodz / Litzmannstadt. In Kooperation mit Julian Baranowski, Joanna Podolska, Krystyna Radziszewska, Jacek Walicki. Unter Mitarbeit von Imke Janssen-Mignon, Andrea Löw, Joanna Ratusinska, Elisabeth Turvold und Ewa Wiatr. 5 Bde. Göttingen. (=Schriftenreihe zur Lodzer Getto-Chronik).

FlatT OSKar (1853): Opis miasta Łodzi. Pod względem historycznym, statystycznym $i$ przemystowym. [Die Beschreibung der Stadt Lodz unter geschichtlichem, statistischem und industriellem Aspekt]. Warszawa [=Nachdruck Łódź 2002]. 
FrejDlich, KonRAD (ed.) (1973): Uśmiech Ariadny. Antologia reportażu Lódzkiego. Od Oskara Flatta do Andrzeja Makowieckiego. [Das Lächeln der Ariadne. Eine Anthologie Lodzer Reportagen. Von Oskar Flatt bis Andrzej Makowiecki]. Łódź.

FrIEDMan, FILIP (1935): Dzieje Żydów w Łodzi od poczqtków osadnictwa Żydów do r. 1863. Stosunki ludnościowe, życie gospodarcze, stosunki spoteczne. [Die Geschichte der Juden in Lodz seit den Anfängen der jüdischen Ansiedelung bis 1863. Bevölkerungsverhältnisse, das wirtschaftliche Leben und soziale Beziehungen]. Łódź.

GEBHARD, JÖRG (ed.) (2006): Lublin. Eine polnische Stadt im Hinterhof der Moderne (1815-1914). Köln/Weimar/Wien.

GeYer, Helena ANNA (2002): Aus meinem Leben. Erinnerungen aus den Jahren 18851914. Z mojego życia. Wspomnienia z lat 1885-1914. Hrsg. von Krzysztof Woźniak. Łódź.

GinSBERT, ADAM (1962): Łódź. Studium monograficzne. [Lodz. Eine monographische Studie]. Łódź.

GÓRECKI, WOJCIECH (1998): Łódź przeżyła katharsis. [Lodz erlebte eine Katharsis]. Łódź.

GUESNET FRANÇOIS (1997): Żydowskie i niemeckie organizacije w Lodzi XIX wieku: typy $i$ stosunki. [Jüdische und deutsche Organisationen im 19. Jh. in Lodz: Typen und Beziehungen]. In: SAMUŚ, 162-191.

- (1997a): Lodzer Juden im 19. Jahrhundert. Ihr Ort in einer multikulturellen Stadtgesellschaft. Leipzig.

- (1998): Polnische Juden im 19. Jahrhundert. Lebensbedingungen, Rechtsnormen und Organisation im Wandel. Köln/Weimar/Wien.

- (1999): „Die beiden Bekenntnisse leben weit entfernt voneinander, sie kennen und schätzen sich gegenseitig nicht. " Das Verhältnis von Juden und Deutschen im Spiegel ihrer Organisationen im Lodz des 19. Jahrhunderts. In: HENSEL, 139-170.

Heiкe, Отто (2002): Leben im deutsch-polnischen Spannungsfeld. Erinnerungen und Einsichten eines deutschen Journalisten aus Lodz. Herne.

HENSEL, JÜRGEN (1996): Konferenzbericht zu „Gruppenbeziehungen in einer multiethnischen Stadtgesellschaft: Polen, Deutsche und Juden in Lodz im 19. und 20. Jahrhundert (bis 1939)“" vom 19.-22. Oktober 1995 in Łódź. In: 2. Bulletin des Deutschen Historischen Instituts Warschau: http://www.dhi.waw.pl/bulletin2/bd2_07.htm (01.9.2006).

- (ed.) (1999): Polen, Deutsche und Juden in Lodz 1820-1939. Eine schwierige Nachbarschaft. Osnabrück.

HePPNer, Harald (2000): Czernowitz. Die Geschichte einer ungewöhnlichen Stadt. Köln/ Weimar/Wien.

Hertz, MieczysŁaw (1933): Łódź w czasie wielkiej wojny. [Lodz während des Großen Krieges]. Łódź.

HIRSCHHAUSEN, UlRIKe vON (2006): Die Grenzen der Gemeinsamkeit. Deutsche, Letten, Russen und Juden in Riga 1860-1914. Göttingen. 
Die Wiederentdeckung der polyethnischen Vergangenheit der Stadt Łódź

HofMANN, ANDREAS R. (2002): Imageprobleme einer Antimetropole. Lodz 1900-1930. In: Hofmann, ANDreas R. / WendLAND, ANNA Veronika (eds.): Stadt und Öffentlichkeit in Ostmitteleuropa 1900-1939. Beiträge zur Entstehung moderner Urbanität zwischen Berlin, Charkiv, Tallinn und Triest. Stuttgart, 235-257.

- (2002a): An Ephemeral Utopia of Nationalities? Lodz in the Great War, 1914-1918. In: Papers of the Sixth International Conference on Urban History of the European Association of Urban History (EAUH), Edinburgh, September 4-7, 2002: http//www. esn.ac.uk/urban_history/text/HofmannM6.doc (1.9.2006).

- (2004): Die vergessene Okkupation. Lodz im Ersten Weltkrieg. In: Löw, ANDREA / Robusch, Kerstin / Walter, STEFAnie (eds.): Deutsche - Juden - Polen. Geschichte einer wechselvollen Beziehung im 20. Jahrhundert. Festschrift für Hubert Schneider. Frankfurt (M.), 59-78.

- (2004a): Reweaving the Urban Fabric: Multiethnicity and Occupation in Łódź, 19141918. In: FunCK, MARCUS / ChICKERING, Roger (eds.): Endangered Cities. Military Power and Urban Societies in the Era of the World Wars. Boston, 81-94.

INDELAK, JACEK (1997): Die Stadt der vier Kulturen und andere Lodzer Mythen. In: Reise nach Lodz. StadtBauwelt. Vierteljahreshefte der Bauwelt 48 vom 26.12.1997: 2716-2721.

JOACHIMSTHALER, JÜRGEN (2002): Zur Einführung. In: [ANONYM], 9-14.

- (2002a): Die Literarisierung einer Region und die Regionalisierung ihrer Literatur. In: [ANONYM], 17-49.

- (2002b): Regionalität als Kategorie der Sprach- und Literaturwissenschaft. In: [ANONYM], 491-501.

- (2007): Philologie der Nachbarschaft. Erinnerungskultur, Literatur und Wissenschaft zwischen Deutschland und Polen. Würzburg.

- (2007a): Die Zukunft der Vergangenheit. Die Auseinandersetzung der polnischen Germanistik mit den deutschen Spuren in Polen. Endgültige Fassung. In: JOACHIMSTHALER, 13-37.

JORDAN, JORDAN (o.J. [2007]): W kręgu tódzkiej secesji. [Im Bereich der Lodzer Sezession]. Łódź.

KAMIŃSKA, MARIA (1997): Językowe wyktadaniki wielonarodowego charakteru Łodzi w miejskiej polszczyźnie mówionej. [Sprachliche Nachweise des multikulturellen Charakters von Lodz in der städtischen gesprochenen Sprache]. In: SAMUŚ, 341-348.

- (1999): Das Lodzer Polnisch als Ausdruck des multiethnischen Charakters der Stadt. In: HENSEL, 301-306.

Kaszubina, WisŁawa (1967): Bibliografia prasy tódzkiej 1863-1944. [Bibliographie der Lodzer Presse 1863-1944]. Warszawa.

Kempa, ANDRZEJ / SzUKALAK, MAREK (2001-2004): Żydzi dawnej Łodzi. Stownik biograficzny żydów tódzkich oraz z Łodziq zwiqzanych. [Die Juden vom alten Lodz. Ein biographisches Wörterbuch der Lodzer Juden]. 4 Bde. Łódź.

- (2006): The Biographical Dictionary of the Jews from Lodz. Łódź. 
Frank M. Schuster

Kessler, Wolfgang (2002): Otto Heike und die Geschichte der Deutschen in Mittelpolen. In: HeIKE, 147-165.

KING, JEREMY (2002): Budweisers into Czechs and Germans. A Local History of Bohemian Politics, 1848-1948. Princeton (NJ)/Oxford.

KopCZYŃSKA-JAWORSKA, BRONISŁAWA (1999): Der Eigene und der Fremde. Formen kultureller Distanz. In: HENSEL, 257-268.

KOSSERT, ANDREAS (2006): ,Gelobtes Land?' Religiosität und Unternehmer in der Industrielandschaft. Lodz und Manchester im Langen 19. Jahrhundert. In: GEBHARD, 143-163.

KosSMAnN, OSKaR (1966): Lodz. Eine historisch-geographische Analyse. Würzburg.

Koter, Marek / Kulesza, Mariusz / Puś, Wiestaw / Pytlas, Stefan (2005): Wyptyw wielonarodowego dziedzictwa kulturowego Łodzi na wspótczesne oblicze miasta. [Der Einfluss des multinationalen kulturellen Erbes von Lodz auf das Antlitz der modernen Stadt]. Łódź.

KowALSKI, WiTOLD (1999): Leksykon łódzkich fabryk. [Lexikon der Lodzer Fabriken]. Łódź.

Krogerus, Mikael (2004): Lodz in Translation. In: Aus dem Osten. Neue Züricher Zeitung Folio 4, 05.4.2004.

KuCNER, Monika (2001): Prasa Niemiecka $w$ Łodzi. [Die deutsche Presse in Lodz]. In: KUCZYŃSKI / RATECKA, 209-234.

- (2005): Prasa Niemiecka $w$ Łodzi w okresie międzywojennym. [Die deutsche Presse zwischen den Kriegen.]. In: KUCZYŃSKI, 161-180.

KuCZYŃSKI, KRZYSZTOF A.: (1999): Czarodziej z Darmstadt. Rzecz o Karlu Dedeciusie. [Der Zauberer aus Darmstadt - über Karl Dedecius]. Łódź.

-(ed.) (2005): Wizerunek Łodzi w literaturze, kulturze $i$ historii Niemiec $i$ Austrii. Materiały z konferencji, która odbyła się w Łodzi w dniach 19-20 października 2005 w ramach obchodów Roku Krajów Obszaru Języka Niemieckego. [Das Bild von Lodz in Literatur, Kultur und Geschichte Deutschlands und Österreichs. Materialien einer Konferenz, veranstaltet vom 19. bis 20. Oktober in Lodz im Rahmen der Feierlichkeiten ,zum Jahr der deutschsprachigen Länder 2005 ‘]. Łódź.

KuCZyŃSKi, KrZysztof A. / BARTOSZEWSKA, IrenA (eds.) (2000): Karl Dedecius: ambasador kultury polskiej w Niemczech. [Karl Dedecius: Botschafter der polnischen Kultur in Deutschland]. Łódź.

KuCZYŃSKi, KrZYSZTOF A. / KuCHARSKA, Dorota (eds.) (1998): Gerda Leber-Hagenau a stosunki polsko-austriackie w XX wieku. [Gerda Leber-Hagenau und die polnisch-österreichischen Beziehungen im 20. Jhd.]. Płock/Toruń.

KuCZyŃSKi, KRZYSZTOF A. / RATECKA, BARBARA (eds.) (2001): Niemcy w dziejach Łodzi do 1945 roku. Zagadnienia wybrane. [Die Deutschen in der Geschichte von Lodz bis 1945. Ausgewählte Probleme]. Łódź.

KuCZyŃSki, KrZYsztof A. / RATECKA, BARbARA (2005): Vorwort. In: KuCZYŃSKi / RATECKA / MEHNERT, 5-10. 
Die Wiederentdeckung der polyethnischen Vergangenheit der Stadt Łódź

KucZyŃski, Krzysztof A. / RATECKA, BARBARA / MEHNERT, ElKe (eds.) (2005): Lodz in der deutschsprachigen Literatur. Eine Anthologie. Łódź.

KuCZYŃSKi, KRZYSZTOF A. / SchNEIDER, LothAR (eds.) (1991): Das literarische Antlitz des Grenzlandes. Frankfurt (M.) u. a.

Kuligowska-KorZeniewska, AnNa (1976): Trudne poczatki. Teatr tódzki w latach 1844-1863. [Schwierige Anfänge. Der Lodzer Theater in den Jahren 1844-1863]. Wrocław.

- (1995): Scena obiecana. Teatr polski w Łodzi 1844-1918. [Die gelobte Bühne. Das polnische Theater in Lodz 1844-1918]. Łódź.

- (1997): Łódź teatralna: polska, niemiecka i żydowska. Wspótpraca i rywalizacja. [Die polnisch-deutsch-jüdische Theaterlandschaft in Lodz. Zusammenarbeit und Rivalität]. In: SAMUŚ, 240-259.

- (1999): Die polnisch-deutsch-jüdische Theaterlandschaft in Lodz. Zusammenarbeit und Rivalität. In: HENSEL, 307-323

Kuligowska-Korzeniewska, AnNA / Leyko, MaŁgorzata (eds.) (1998): Teatr żydowski w Polsce. Materiaty z międzynarodowej konferencji naukowej. [Das jüdische Theater in Polen. Materialien der internationalen Wissenschaftskonferenz]. Łódź.

KuźKo, WANDA (2000): Biedermannowie. Dzieje rodziny i fortuny 1730-1945. [Die Biedermanns. Die Geschichte einer Familie und ihres Vermögens]. Łódź.

Leyko, Matgorzata (1992): Das jüdische Theater in Lodz, Stadt dreier Nationen. In: BAYERDÖRFER, HANS-PETER (ed.): Theatralia Judaica. Emanzipation und Antisemitismus als Momente der Theatergeschichte. Von der Lessing-Zeit bis zur Shoah. Tübingen, 218-228.

- (ed.) (2000): Sceny żydowskie. Studia i materiaty. [Jüdische Bühnen. Studien und Materialien]. Łódź.

LINDNER, RAINER / PIETROW-ENNKER, BIANKA (eds.): Unternehmer im Russischen Reich. Sozialprofil, Symbolwelten, Integrationsstrategien im 19. und frühen 20. Jahrhundert. Osnabrück, 143-163.

LindNeR, Rolf (2004): Offenheit - Vielfalt - Gestalt. Die Stadt als kultureller Raum. In: JAEGER, FRIEDRICH / RÜSEN, JÖRN (eds.): Handbuch der Kulturwissenschaften. Bd. 3: Themen und Tendenzen. Stuttgart/Weimar, 385-398.

Löw, ANDREA (2006): Juden im Getto Litzmannstadt. Lebensbedingungen, Selbstwahrnehmung, Verhalten. Göttingen.

Loew, Peter Oliver (2003): Danzig und seine Vergangenheit 1793-1997. Die Geschichtskultur einer Stadt zwischen Deutschland und Polen. Osnabrück.

LUNZER, RENATE (2002): Triest. Eine italienisch-österreichische Dialektik. Klagenfurt. MACHEJEK, ANDRZEJ (ed.) (2004): Żydzi Łódzcy. Jews of Łódź. Łódź.

- (ed.) (2005): Niemcy Łódzcy. Die Lodzer Deutschen. Łódź.

Magris, Claudio / ARA, Angelo ( $\left.{ }^{2} 2005\right)$ : Triest. Eine literarische Hauptstadt in Mitteleuropa. München. 
Mensching, StefFen (2006): Ohne Theo nach Lodz. In: Mensching, StefFEn: Ohne Theo nach Lodz und andere Reisegeschichten. Berlin, 13-20.

MiChOŃ, MARCIN (2005): Die Textsorte „Kommentar“ in der Lodzer Zeitung. In: RIEKE, JÖRg / SChUSTER, BritT-MARIE (eds.): Deutschsprachige Zeitungen in Mittelund Osteuropa. Sprachliche Gestalt, historische Einbettung und kulturelle Traditionen. Berlin, 251-266.

Mostowicz, ARnold (1988): Żótta gwiazda i czerwony krzyż. [Gelber Stern und Rotes Kreuz]. Warszawa. Deutsch unter dem Titel: BodeK, ANDRZEJ (ed.) (1992): Der Blinde Maks oder der Passierschein durch den Styx. Übersetzt von Karin Wolff und Andrzej Bodek. Berlin.

NASARSKi, Peter E. (ed.) (1988): Lodz. „Gelobtes Land.“ Von deutscher Tuchmachersiedlung zur Textilmetropole im Osten. Dokumente und Erinnerungen. Berlin/Bonn.

NeumanN, BiRGit (2005): Erinnerung - Identität - Narration. Gattungstypologie und Funktionen kanadischer ,Fictions of Memory“. Berlin/New York.

- (2005a): Literatur, Erinnerung, Identität. In: ERLL / NüNNING, 149-178.

PeŁKA, ARtur (1994): Dyrekcia Adolfa Kleina $w$ teatrze niemieckim Thalia $w$ Lodzi (1909-1914). [Die Direktion Adolf Kleins im deutschen Thalia-Theater In Lodz (19091914).] Unveröffentlichte Magisterarbeit. Łódź.

- (2005): Das deutsche Theater in der Dreivölkerstadt Lodz - Die Direktion Adolf Kleins am Thalia Theater (1909-1914). In: FASSEL, HoRst / LeYKo, MAŁgORZATA / Ulrich, Paul S. (eds.): Polen und Europa. Deutschsprachiges Theater in Polen und deutsches Minderheitentheater in Europa. Łódź (=Thalia Germanika 6).

Pietrow-EnNKer, BiAnKA (1995): Brennspiegel Lodz: Zur Problematik gesellschaftlicher Modernisierung im Königreich Polen (1820-1914). In: Kulturwart. Beiträge zur deutsch-polnischen Nachbarschaft 199:1-13, 200:17-25.

- (1999): Auf dem Weg zur Bürgergesellschaft? Modernisierungsprozesse in Lodz (1820-1914). In: HENSEL, 103-130.

- (2002): Der „Lodzermensch“ verkörpert eine Lebensweise, die in Lodz wieder modern wird. In: Frankfurter Allgemeine Zeitung, 03.1.2002.

- (2002a): Unternehmer und Öffentlichkeit: Kommunikation und Symbolwelt von Wirtschaftsbürgern in Städten des westlichen Zarenreiches 1860-1914. In: KultuRWISSENSCHAFTLICHES FORSCHUNGSKOLLEG / SONDERFORSCHUNGSBEREICH 485: Norm und Symbol. Die kulturelle Dimension sozialer und politischer Integration. Ergebnisbericht. Konstanz, 391-430.

- (2003): Wirtschaftsbürger und „Bürgerlichkeit“: zur Konstituierung des Unternehmertums in Städten des westlichen Zarenreiches (19./20. Jh.). In: NIETYKSZA, MARIA et al. (eds.): Spoteczeństwo w dobie przemian: wiek XIX $i$ XX. Ksiega jubileuszowa profesor Anny Żarnowskiej. Warszawa, 257-266.

- (2005): Wirtschaftsbürger und Bürgerlichkeit im Königreich Polen: Das Beispiel von Lodz, dem „Manchester des Ostens“. In: Geschichte und Gesellschaft 2:169-202.

PODOLSKA, JoANNA (2004): Żótta ksiażka Łódź. Łódź. 
Die Wiederentdeckung der polyethnischen Vergangenheit der Stadt Łódź

- (2005): Das Goldene Buch Łódź. Übersetzt von Maria Goldstein und Annette Zaborowski. Łódź.

- (2006): The Golden Book of Łódź. Übersetzt von Alicja Weikop. Łódź.

Polcuch, VAlENTIN (1975): „Theo, wir fahr'n nach Lodz“. Alter Lodz-Song wurde bundesdeutscher Schlager Nr. 1. In: Jahrbuch Weichsel-Warthe:112-114.

Polonsky, Antony (ed.) (1991): Jews in Lodz 1820-1939. In: Polin 6:1-287.

Prykowska-Michalak, Karolina (2005): Teatr niemiecki $w$ Łodzi. Szeny, wykonawcy, repertuar (1867-1939). [Das deutsche Theater in Lodz. Inszenierungen, Mitwirkende und Repertoirs]. Łódź.

Puś, WiESŁAW (1998 / 2001): Żydzi w Łodzi w latach zaborów 1793-1914. [Die Juden in Lodz in den Jahren der Teilung 1793-1914]. Łódź.

Puś, WiesŁaw / Liszewski, StanisŁaw (eds.) (1991): Dzieje Żydow w Łodzi 18201944. Wybrane problemy. [Die Geschichte der Juden in Lodz 1820-1944. Ausgewählte Probleme]. Łódź.

Pytlas, Stefan (1994): Łódzka burżuazja przemystowa $w$ latach 1864-1914. [Das industrielle Bürgertum in Lodz in den Jahren 1864-1914]. Łódź.

RABON, IsRAEL (1928 / 1986): Di Gas. Varshe / Jerusalem.

- (1934): Balut. [Bałuty]. Varshe.

- (1998): Die Straße. Übersetzt von Thomas Soxberger. Salzburg/Wien.

RADA, UwE (2001): Warten auf Mr. Bloomfield. In: Berliner Zeitung, 21.4.2001:M06P.

RADZISZEWSKA, KRYSTYNA (ed.) (1997 / 22001): Niemieckimi śladami po ,Ziemi Obiecanej '. Auf deutschen Spuren im ,Gelobten Land'. Łódź.

- (ed.) (1999): Gdzie sq Niemcy z tamtych lat? - Wspomnienia łódzkich Niemców. Sag mir, wo die Deutschen sind? - Erinnerungen der Łódźer Deutschen. Łódź.

- (ed.) (2002): Tonqca Łódź (lata 1939-1945). Das sinkende Boot (der Zeitraum 19391945 [sic]). Łódź.

- (2005): Die Lodzer Presselandschaft. In: RIEKE / SCHUSTER , 213-223.

RADZISZEWSKA, KRYSTYNA / RIECKE, JÖRG (eds.) (2004): Germanizacja Lodzi $w$ nazistowskiej prasie z lat 1939-1943 - wybór artykułów. Die Germanisierung von Lodz im Spiegel der nationalsozialistischen Presse (1939-1943). Łódź.

RADZISZEWSKA, KRYSTYNA / WoźNIAK, KRZYSZTOF (2000): Pod jednym dachem. Niemcy oraz ich polscy $i$ żydowscy sqsiedzi $w$ Lodzi XIX $i$ XX wieku. Unter einem Dach. Die Deutschen und ihre polnischen und jüdischen Nachbarn in Lodz im 19. und 20. Jahrhundert. Łódź.

RAHDEN, TILl VAN (2000): Juden und andere Breslauer. Die Beziehungen zwischen Juden, Protestanten und Katholiken in einer deutschen Großstadt von 1860 bis 1925. Göttingen.

RENNER, AlFRED (1983): Deutsche Schicksalswege in Lodz. Ein historischer Roman. Berlin/ Bonn. 
Frank M. Schuster

Reymont, WŁadysŁaw StanisŁaw (1899 / 1970): Ziemia obiecana. [Das gelobte Land]. 2 Bde. Warszawa.

- (1914 / $\left.{ }^{10} 1917\right)$ : Das gelobte Land. Übersetzt von Aleksander von Guttry. München.

- (1984): Das gelobte Land. Übersetzt von Aleksander von Guttry und Sigrid Moser. Leipzig.

RIEKE, JÖRg / Schuster, BRITT-MARIE (eds.): Deutschsprachige Zeitungen in Mittelund Osteuropa. Sprachliche Gestalt, historische Einbettung und kulturelle Traditionen. Berlin.

Rosin, RyszARD / BARANOwSKi, BoHdAn, FiJAŁEK, JAN (eds.) (1980 / ${ }^{2} 1988$ ): Łódź. Dzieje Miasta. Tom I. Do 1918 r. [Lodz. Stadtgeschichte. Bd. 1. Bis 1918]. Warszawa/Łódź.

Rosin, Ryszard / SPYCHALSKI, JóZEF et al. (eds.) (1966): Wtókniarze tódzcy. Monografia. [Der Lodzer Textilarbeiter. Eine Monographie]. Łódź.

Rosset, Edward (1929): Łódź. Miasto Pracy. [Lodz. Stadt der Arbeit]. Łódź.

Roth, JosePH (1928): Russische Überreste - Die Textilindustrie in Lodz. In: Frankfurter Zeitung, 19.7.1928. Wieder gedruckt in: ROTH, JOSEPH (1994): Briefe aus Polen. In: Werke in 6 Bänden. Hrsg. von Fritz Hackert und Klaus Westermann. Bd. 2: Das journalistische Werk. Hrsg. von Klaus Westermann. Frankfurt (M.)/Wien, 935-970, hier 949-953.

Rubinstein, ARTUR (1973): My Young Years. New York.

SADZIŃSKI, RoMAn (2005): Die Sprache der Lodzer Deutschen und der Lodzer Zeitung. In: RIEKE / SCHUSTER, 279-285.

SAmuŚ, Pawet (ed.) (1997): Polacy - Niemcy - Żydzi w Lodzi w XIX-XX w. Sasiedzi dalecy $i$ bliscy. [Polen, Deutsche und Juden im 19. und 20. Jhd. in Lodz. Nahe und entfernte Nachbarn]. Łódź.

SAmuś, PaweŁ / Puś, WiesŁaw (eds.) (2006): Fenomen getta tódzkiego 1940-1944. [Das Phänomen Lodzer Ghetto1940-1944]. Łódź.

SCHLÖGEL, KARL (1996): Lodz - Suche nach dem ,Gelobten Land'. In: Die Zeit, 13.9.1996. Wieder gedruckt in: SCHLÖGEL, KARL (2003): Promenade in Jalta und andere Städtebilder. Frankfurt (M.), 126-138.

Schmidt, Sabine / Dewertmann, Franz / Rusak, Elżbieta (eds.) (1993): Oskar Tulla - Mahlke ... : śladami gdańskich bohaterów Güntera Grassa. [Oskar - Tulla Mahlke ... Auf den Spuren der Danziger Helden bei Günter Grass]. In Gdańsk unterwegs mit Günter Grass. Gdańsk.

SCHUSTER, BRITT-MARIE (2005): Die Entwicklung von Nachrichtentexten (1873-1914) in der Lodzer Zeitung im Vergleich mit binnendeutschen Zeitungen (Gießener Anzeiger und Frankfurter Zeitung). In: RIEKE / SCHUSTER, 225-249.

SCHUSTER, FrAnK M. (2004): Zwischen allen Fronten. Osteuropäische Juden während des Ersten Weltkrieges (1914-1919). Köln/Weimar/Wien. 
Die Wiederentdeckung der polyethnischen Vergangenheit der Stadt Łódź

- (2007): Fiktion und Wirklichkeit - Wirklichkeit als Fiktion in Joseph Roths Roman „Hotel Savoy“. In: FeuCHERT, SASCha / JABŁKOwSKA, JOANNA / RIEKE, JÖRG (eds.): Literatur und Geschichte. Festschrift für Erwin Leibfried. Frankfurt (M.) u. a., 185-197.

- (2007a): Zwischen Identitätskrise und Herausforderung: Polen, Juden, Deutsche während des Ersten Weltkrieges in der Textilmetropole Lodz. In: LASATOWICZ, MARIA KATARZYNA / [RUDOLPH, ANDREA] (eds.): Städtische Räume als kulturelle Identitätsstrukturen. Schlesien und andere Vergleichsregionen. Berlin, 81-95.

- (2007b): Zwischen Hoffen und Bangen. Reaktionen jüdischer Literaten auf das ,Wunder der Wiedergeburt' Polens 1918-1921. In: Nordost-Archiv XVI [im Druck].

SCHUSTER, FRANK M. / CAYSA, VOLKER (2006): Vom Vergessen per Erinnerung. Über ein in der deutschen Kriegserinnerung vergessenes Kriegsereignis. Zapominanie przez pamięć. $O$ wydarzeniu wojennym, które zostało zapomniane przez niemiecka pamięć historycznq. In: WERNER, PIOTR (ed.): Wielka Wojna o Ziemię Obiecanq. Operacja Łódzka 1914. Praca zbiorowa. Łódź, 77-89.

SHPIGL, Yesheyhu (1966): Shtign tsum himl. [Die Himmelsleiter]. Tel-Aviv.

- (1990): Drabina do nieba. [Die Himmelsleiter.] Übersetzt aus dem Französischen von Anna Osmólska-Mętrak. Warszawa.

SINGER, IsRAEl JoShUA (1936): Di brider Ashkenazi. New York.

- (1997): Die Brüder Aschkenasi. Übersetzt aus dem Amerikanischen von Gertrud Baruch. Frankfurt (M.).

SINGER, OSKAR (2002): „Im Eilschritt durch den Getto-Tag“. Reportagen und Essays aus dem Getto Lodz 1942-1944. Hrsg. von Sascha Feuchert, Erwin Leibfried, Jörg Riecke, Julian Baranowski, Krystyna Radziszewska, Krzysztof Wozniak. Berlin.

- (2002): Przemierzajac szybkim krokiem getto... Reportaże i eseje z getta tódzkiego. Übersetzt von Krystyna Radziszewska. Łódź.

SKIBIŃSKI, ZIEMOWIT / STELMASZCZYK-ŚwIONTEK, BARBARA (eds.) (1982): Kwiaty Łódzkie. Antologia poezji o Łódzi. [Lodzer Blumen. Eine Anthologie der Lyrik über Lodz]. Łódź.

SKRZYDŁO, LESZEK (1999): Rody fabrykanckie. [Fabrikantenfamilien]. Łódź.

- (2000): Rody fabrykanckie. [Fabrikantenfamilien]. 2 Bde. Łódź.

- (2007): Rody nie tylko fabrykanckie. [Nicht nur Fabrikantenfamilien]. Łódź.

SPODENKIEWICZ, PAWEe (1998): Zaginiona dzielnica. Łódź żydowska-ludzie i miejsca. [Der verschwundene Stadtteil. Das jüdische Lodz. Menschen und Orte]. Łódź.

- (2007): The Missing District. People and Places of Jewish Łódź. Übersetzt von Dorota Wiśniewska und John Crust. Łódź.

STEFAŃSKI, KRZYSZTOF (2003): Atlas Architektury dawnej Łodzi do 1939 r. [Architekturatlas des alten Lodz bis 1939]. Łódź.

- (2005): Piotrkowska 77. Łódź.

- (2006): Willa Oskara Zieglera. Siedziba Łódzkiego Towarzystwa Naukowego. [Die Oskar-Ziegler-Villa. Sitz der Lodzer Wissenschaftlichen Vereinigung]. Łódź. 
Frank M. Schuster

Steinhagen, Eliza Maria (2003): Kruschowie. Historia rodu. [Die Krusches. Geschichte einer Familie]. Łódź.

STOLARZEWCZ, LUDWIK (1935): Literatura Łodzi w ciagu jej istnienia. Szkic literacki $i$ antologia. [Die Literatur über Lodz in der Zeit ihrer Existenz. Essay und Anthologie]. Łódź.

Strobel, GEORG W. $\left({ }^{3} 1993\right)$ : Lodz eine Vielvölkerstadt Polens. In: KobYlińSKA, Ewa / Lawaty, Andreas / Stephan, RüDiger (eds.): Deutsche und Polen. 100 Schlüsselbegriffe. München/Zürich, 508-514.

StÜBEn, JENS (2002): ,Regionale Literatur' und, Literatur der Region`. Zum Gegenstandsbereich einer Geschichte der deutschen Literatur in den Kulturlandschaften Ostmitteleuropas. In: [ANONYM], 51-75.

SzukalaK, MareK (1990): Miasta Lodzermenschów. [Die Stadt der Lodzermenschen]. Łódź.

- (2007): Stownik biograficzny żydów tódzkich oraz z Łodziq zwiazanych. Seria II. [Biographisches Wörterbuch der Lodzer Juden]. Bd. 1. Łódź.

TEPLITZKA, BerTA (1913): Lodzer Typen. Lodz.

- (2008): Lodzer Typen. Portrety Łódzkie. Hrsg. von Małgorzata Półrola. Łódz.

Thum, Gregor (2003): Die fremde Stadt. Breslau 1945. Berlin.

Treger, MAX (1985): Lodz - Berlin 1914-1984. Ein Zeitdokument. Frankfurt (M.).

Trunk, Yehiel Yeshaia (1944-1953): Poiln. [Polen]. Bde. I-VII. New York.

WALICKI, JACEK (2000): Synagogi $i$ domy modlitwy $w$ Lodzi (do 1939 r.). The Synagogues and Prayer Houses of Łódź (to 1939). Übersetzt von Guy Russel Torr. Łódź.

WEIGT, ZENON (2005): Einblicke in die Anzeigen der Lodzer Zeitung. In: RIEKE / SCHUSTER, 267-278.

Wilk, MaRian (1996): Niemcy w Łodzi do 1939 roku. [Die Deutschen in Lodz bis 1939]. Łódź.

WiLMAŃSKI, JeRZY (1993): Ptatki sadzy. Antologia felietonistyki łódzkiej. [Rußflocken. Eine Anthologie der Lodzer Feuilletonautoren]. Łódź.

ZeH, Juli (2003): Dann fahr doch! In: Die Zeit, 06.3.2003 [Reisebeilage]. 